\title{
A Dynamic Monitoring Framework and Methodologies Adapted for Grid GARUDA
}

\author{
Divya $\mathbf{M G}^{a}$, Santhosh $\mathbf{J}^{a}$, HenrySukumar ${ }^{a}$, GnanaSeelan $\mathbf{M}^{b}$, Prasanna $\mathbf{P}^{c}$, \\ Subrata Chattopdhyay ${ }^{a}$ \\ Centre for Development of Advanced Computing \\ ${ }^{a}$ Bangalore, ${ }^{b}$ Hyderabad, ${ }^{c}$ Chennai, India \\ E-mail: (divyam, santhoshj, henrys, mgseelan, prasannap, subratac) @cdac.in
}

\begin{abstract}
This paper highlights grid operational processes, which are being adapted and practiced to monitor and manage the Indian national grid computing initiative GARUDA. GARUDA is an established grid, spread across more than 30 Indian cities and connected to more than 70 institutes. Various Virtual Organizations (VO) are formed for user communities to collaborate for the realization of common goals. However the resources in the grid may dynamically join or leave and may vary with respects to their administration membership. In this transient nature, the users must be able to keep track of resources and the grid operations are required to monitor and manage the shared resources (software, hardware, data and instruments) for efficient scheduling, data replication, accounting, performance analysis and optimization of resources. To achieve this, various open source and in-house developed tools are used.

Monitoring broadly includes network health, cluster up time, middleware and related service status, host certification status, grid security, job statistics and analysis, resources status and so on. Problems are accordingly attended and based on their nature they are escalated to concerned teams, the National Knowledge Network (NKN) team and resource provider institute's Grid Administrators (GA). Various tools that are used for grid monitoring and operation are Paryavekshanam, Nagios, Link Monitoring Tool, Request Tracker etc. Most of the component failures are reported through alert emails to GA. Online reports are available for ease operation. Test scripts are installed at GARUDA Head Node (GHN) for automatic restarting of non functional components. All the results can be viewed online and data archiving is implemented for old data. We have found that proper monitoring methodology, highly transparent and interactive work culture and adhering to quality standards*gives good results in providing high grid availability and stability.
\end{abstract}

The International Symposium on Grids and Clouds (ISGC) 2013,

March 17-22, 2013

Academia Sinica, Taipei, Taiwan

*http://www.iso.org/ (ISO 9001:2008) 


\section{Introduction}

Grid monitoring and operation processes should be designed to address all aspects of grid health. A key factor for grid health and reliability is co ordination between Grid Administrators (GA) at every resource provider institute and highly structured transparent work methodology. The grid environment should provide a mechanism for real-time monitoring and notification [1]. The basic purpose of grid monitoring is to ensure that all the components of the grid system are functional and operate in an optimal manner. It is essential that failures of critical components are detected and rectified promptly [2].

This paper is organized as follows, Sections 2 provides a broad view of grid monitoring and operational activities. Section 3 shows the GARUDA grid architecture, network, the partners etc. Section 4 gives information about tools that are used at GARUDA for monitoring. In section 5 , operational challenges are discussed. In section 6 , the monitoring and operational activities are represented. Section 7 explains the importance of meetings and workshops. The section 8 concludes the paper.

\section{Grid Monitoring and Operational Activities}

Computational and Data grids aggregate resources that are geographically spread. These grids are often large highly distributed and have multiple components to monitor and manage. Some components require constant monitoring, while others need to be monitored only on demand. The objective of monitoring is to provide stable services with high availability. Apart from monitoring if there is any scheduled maintenance at any cluster it should be announced to user communities and concerned GA well in advance, so that user can plan their job scheduling accordingly. The major monitoring and operational activities are defined in the following sections.

\subsection{Link Health Check}

Link healthiness is one of the most critical and important requirements as groups of cluster are connected through a high speed network. Monitoring the health of links, providing redundancy and detection of link failure are essential. Link health checks should include all network parameters like latency, packet loss, CPU utilization, bandwidth input and output.

\subsection{Grid Head Node Health Check}

Grid head nodes to be monitored continuously at a predefined rate for their reachability and availability. Other aspects to be monitored are login or access to these nodes, load on cluster, memory, storage and the validity of host certificates.

\subsection{Grid Service Health Check}

The major grid components of GARUDA are Globus Toolkit ${ }^{1}$, Gridway meta scheduler and local resource manager such as PBS, Loadleveler etc. Periodically health check of GSISSH, container, GridFTP, MyProxy, VOMS, Nagios, Ganglia services to be done. Upon failure of any of these services, they are started automatically. For this test scripts should be designed and deployed.

\footnotetext{
${ }^{1}$ http://www.globus.org/
} 


\subsection{Grid Security Check}

All actions that change the state of the grid like maintenance of user lists, granting and revoking of credentials, issuance and updating of certificate revocation lists (CRLs), grid authorization mapping at resource sites, user access to the credential management service, access to GHN and clusters need to be recorded. All these recorded logs are analyzed on day to day basis.

\subsection{Job Statistics and Analysis}

All the important events of the computing environment need to be identified and tracked. Examples of important events include job submission, completed jobs, failed access attempts and resource allocation failures. All pending and failed job logs are analyzed. Grid usage statistics like number of active users who performed job submission, the number of jobs submitted to grid and the respective grid head nodes, CPU hours utilized, the number of CPUs utilized by every job etc are monitored and recorded. These statistics also help in estimating future resource requirements and determining future utilization trends.

\section{Grid Monitoring Tools at GARUDA}

GARUDA is monitored using various tools, services and test scripts. This involves open sources tools such as Nagios and inhouse developed tools like Paryavekshanam, Hemon etc. User support requests are managed using Request Tracker tracking system. Most of the grid component failures are reported through alert emails to GA. Online reports are available for ease operation. Test scripts are installed at GHN for automatic recovery of non functional components. All the results will be accessible online and archiving is possible.

\section{About GARUDA}

GARUDA [3] is the Indian national grid computing initiative. GARUDA stands for Global Access to Resources Using Distributed Architecture. GARUDA is a cluster of clusters. Clusters are heterogeneous in nature and spread across India. As per Ian Foster's three point check list "Grid coordinates resources that are not subject to centralized control" [4], GARUDA has no common centralized control. GARUDA resources are aggregated by partners and C-DAC [5]. The computational and storage resources are aggregated by the Indian research institutes, academic institutes and C-DAC. The resources are located at Ahmedabad, Bangalore, Chennai, Delhi, Guwahati, Hyderabad, Kharagpur and Pune. All the resources are connected to GARUDA through the high speed National Knowledge Network (NKN) [6]. The architecture of NKN has been designed for reliability, availability and scalability. The network consists of an ultra-high speed core infrastructure of links at $2.5 / 10 \mathrm{~Gb} / \mathrm{s}$ and which will be progressively upgraded to $40 / 100 \mathrm{~Gb} / \mathrm{s}$. The core is complemented by a distribution layer covering all districts at appropriate speeds. The participating institutions at the edge would seamlessly connect to NKN at gigabit speed. The NKN shall be a critical information infrastructure for India to evolve as a knowledge society. NKN is a significant step which will enable scientists, researchers and students from across the country to work together for advancing human development in critical and emerging areas. 
The GARUDA can be used only by its partners. There are two types of partnership namely: resource provider and non resources provider. Users who belong to a partner institute can use GARUDA through a valid grid user certificate issued by Indian Grid Certification Authority (IGCA) [7]. For ease of access, many grid access mechanisms are provided. Users can access GARUDA resources either through the NKN network or through Internet.

\section{Grid Monitoring Tools at GARUDA}

GARUDA is monitored using various tools, services and test scripts. This involves open sources tools such as Nagios and inhouse developed tools like Paryavekshanam, Hemon etc. User support requests are managed using Request Tracker tracking system. Most of the grid component failures are reported through alert emails to GA. Online reports are available for ease operation. Test scripts are installed at GHN for automatic recovery of non functional components. All the results will be accessible online and archiving is possible.

\subsection{Grid Monitoring Paryavekshanam}

Paryavekshanam [8] is the GARUDA monitoring tool that can detect any degradation of non functional components so that faults can be fixed at an early stage. It monitors all the important components of grid such as compute resources, network and storage. It is a web based user friendly tool which enhances the reliability, usability and manageability of the infrastructure. It is coined from Sanskrit word which means monitor remotely. The main features are birds eye view of the grid health through Radar Graph, a dash board providing top level views, alerts generation through emails, an easy interface for the addition of new sites, the visualization of data in tabular and graphical format, a data gallery for the analysis of historical data, a search facility for resources and reports, and a facility to see jobs and resource details. It provides the information through services such as the centralized information service, accounting service, and Virtual Organization Management Service (VOMS) [9]. It can generate online reports, supports search facilities and provides a mobile interface. The job statistics report from Paryavekshanam is shown in Figure 1

\subsection{Infrastructure Monitoring Nagios}

Nagios is a powerful monitoring system that enables organizations to identify and resolve Information Technology (IT) infrastructure problems before they affect critical business processes. It detects and repairs problems and mitigates future issues before they affect end-users and customers. It is installed and configured to monitor the entire GARUDA infrastructure [10]. It generates online reports both in table and graphical format. On failure of any system it generates alert emails.

\subsection{Help Desk-Request Tracker (RT)}

RT [11] is the leading enterprise-grade open source issue tracking system. It is installed [12] and configured for bug tracking, help desk ticketing, customer service and workflow processes. Every reported problem is identified as a Ticket. Tickets are classified as minor, major and regular. The criteria for classification depend on the type of incident reported such as unscheduled jobs, service failure, issues with login service, Indian Grid Certification Authority query, a document 


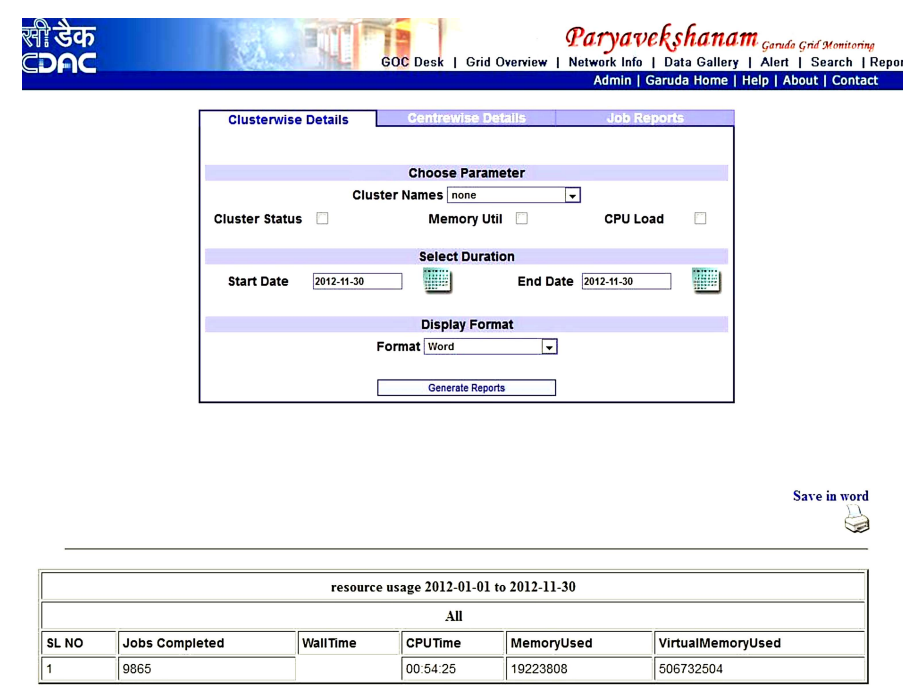

Figure 1: Job statistics from Paryavekshanam

request is an example of a minor ticket. The ticket related to account creation, job submission, software installation etc is a major ticket. Tickets related to installation, configuration, mounting etc at a remote site are classified as regular tickets.

\subsection{Network Monitoring-Hemon}

Hemon [13] is a network monitoring tool developed at C-DAC. This tool monitors link status of resources, which includes availability, latency, packet loss, bandwidth and CPU utilization. Results are displayed as shown in Figure 2. Link status can be queried for the selected duration. Reports can be viewed graphically or in tabular format. Results are available in both percentage and hours.

\begin{tabular}{|c|c|c|c|c|c|c|c|c|}
\hline \multicolumn{9}{|c|}{ Last update of this page on "Tue Feb 28 17:02:19 IST 2012" } \\
\hline S.No. & Location & IP Address & Status & Latency & PKT Loss & CPU Util & BN In & BNW Out \\
\hline 1) & AHM_IPR & 10.118.247.62 & (1) & $39.874 \mathrm{~ms}$ & $0.0 \%$ & $1.0 \%$ & $0 \mathrm{~b} / \mathrm{s}$ & $\mathrm{ob} / \mathrm{s}$ \\
\hline 2) & AHM_PRL & 10.118.247.63 & 包 & $39.979 \mathrm{~ms}$ & $0.0 \%$ & $1.0 \%$ & $3.12 \mathrm{~Kb} / \mathrm{s}$ & $639 \mathrm{Kb/s}$ \\
\hline 3) & AHM_SAC & 10.118.247.61 & (0) & $37.954 \mathrm{~ms}$ & $0.0 \%$ & $1.0 \%$ & $\mathrm{ob} / \mathrm{s}$ & $0 \mathrm{~b} / \mathrm{s}$ \\
\hline 4) & ALH_HCRI & 10.118.247.64 & (2) & NA & $\mathrm{NA}$ & $\mathrm{NA}$ & $\mathrm{NA}$ & $\mathrm{NA}$ \\
\hline 5) & ALH_MNIT & 10.118.247.65 & 四 & $66.221 \mathrm{~ms}$ & $0.0 \%$ & $1.0 \%$ & $0 \mathrm{~b} / \mathrm{s}$ & $0 \mathrm{~b} / \mathrm{s}$ \\
\hline 6) & BLR_CDAC_EC & 10.118.247.69 & (v) & $1.996 \mathrm{~ms}$ & $0.0 \%$ & $1.0 \%$ & $126.62 \mathrm{~b} / \mathrm{s}$ & $174.16 \mathrm{~b} / \mathrm{s}$ \\
\hline
\end{tabular}

Figure 2: GARUDA Link Status from Hemon

\section{Operational Challenges}

In GARUDA the resources are heterogeneous in nature and spread across India. GARUDA resources are aggregated by partners and C-DAC. Maintaining grid availability and reliability is highly challenging in a federated environment. Some of the challenges to mention are: remote ssh access, sudden cluster down time, faster support to users, the provisioning of large home areas for user, lack of cooperation from remote system administrators, the consolidation of expertise of system administrators for better support. 


\section{Sequence of Monitoring Activities at GARUDA}

This section illustrates various components being monitored at GARUDA and the corresponding workflow in Figure 3. As explained in section 4, various tools are used to monitor these components.

The work flow is streamlined as follows: link health is checked if link is healthy and functional the GARUDA resource at remote site can be accessed. Once the resource is accessible the login credentials are checked. After logging in to cluster the load and host certificate validity period are checked. Then health of services like GSISSH, container etc are checked. Using the log files the system access details are verified. Job status are checked, if any job is in pending or queued state analysis is done. Also grid utilization is analyzed like total jobs submitted, number of users used the grid and bandwidth utilized.

If a fault component is detected affecting link health, cluster health, grid login, load on each cluster, host certificate expiry, grid services, grid security etc an action is taken to solve the problem.

\section{Meetings and workshops}

For grid high availability and stability good coordination between GAs is essential. To review GARUDA status and to address unresolved issues every week at a fixed day and time a meeting is conducted with the relevant partners. Once a year a GAs workshop is conducted to share experience and to learn new tools.

\section{Conclusions}

In this paper we have explained how GARUDA is being monitored. Parameters like network health, cluster up time, middleware services status, host certification status, resource usage are regularly monitored. Accordingly problems are attended and based on nature of problem escalated to the deployment team, the network operation team, the national knowledge network team and resource provider institutes grid administrators. We have found that proper monitoring methodology, highly transparent and interactive work culture and adhering to quality standards gives good results in providing high grid availability. There is a need for grid monitoring standards and framework. 


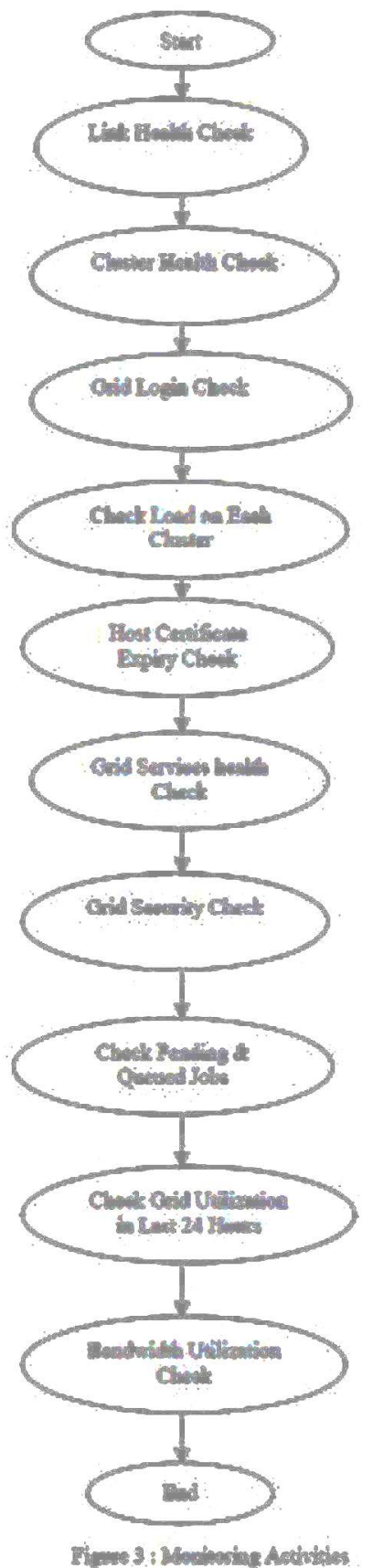

Figure 3: Grid monitoring work flow 


\section{Acknowledgment}

We thank DeitY, India and C-DAC, Bangalore, India. The GARUDA project is funded by Department of Electronics and Information Technology (DeitY). GARUDA is designed, developed and managed by Centre for Development of Advanced Computing (C-DAC)

\section{References}

[1] Ahmer Abbas, Grid Computing A Practical guide to technology and Applications, pp.303-306, 2008

[2] Paryavekshanam, http://gmon.garudaindia.in

[3] GARUDA India web site, http://www.garudaindia.in

[4] Ian Foster and Carl Kesselman, GRID2: Blue print for a new computing infrastructure, Morgan Kaufmann Publishers, pp. 333- 336, 2006

[5] Center for Development of Advanced Computing, http://www.cdac.in

[6] National Knowledge Network, http://nkn.in/garudaoverview.php

[7] Indian Grid Certification Authority, http://ca.garudaindia.in

[8] Karuna, Deepika H.V, Mangala N., Prahlada Rao BB, MohanRam N., Paryavekshanam: A Status Monitoring Tool for India Grid GARUDA, The 24th NORDUnet Conference, Espoo, Finland, 9-11 April 2008

[9] Virtual Organization Membership Service, http://www.globus.org/grid_software/security/voms.php

[10] Nagios Monitoring Tool, http://www.nagios.org/about/overview

[11] Request Tracker, http://bestpractical.com/rt

[12] GARUDA Help desk Support, https://gridsupport.garudaindia.in

[13] Link Monitoring Tool, http://hemon.cdacb.in

[14] Ramesh Naidu Laveti, S. Janakiraman, Mohit Ved and B. B. Prahlada Rao., Seasonal forecast modeling application on the GARUDA Grid infrastructure, The International Symposium on Grids and Clouds (ISGC) 2012, Academia Sinica, Taipei, Taiwan, February 26 âĂŞ March 2, 2012

[15] Pawl Plaszczak and Richard Wellner, Grid Computing-The Savvy Manager Guide, Morgan Kaufmann Publishers, pp. 173-178, 2006 